\title{
CALIDAD DE GRANO Y DE TORTILLAS DE MAÍCES CRIOLLOS DEL ALTIPLANO Y VALLE DEL MEZQUITAL, MÉXICO
}

\section{QUALITY OF GRAIN AND TORTILLA MADE WITH LOCAL MAIZE LANDRACES FROM THE HIGH VALLEYS AND THE VALLEY OF MEZQUITAL, MÉXICO}

\author{
Ma. Gricelda Vázquez Carrillo ${ }^{1 *}$, Juan P. Pérez \\ Camarillo $^{2}$, Juan M. Hernández Casillas ${ }^{3}$, Ma. de \\ la Luz Marrufo Diaz ${ }^{1}$ y Elisa Martínez Ruiz ${ }^{2}$
}

\begin{abstract}
${ }^{1}$ Laboratorio de Calidad de Maíz, Campo Experimental Valle de México, Instituto Nacional de Investigaciones Forestales, Agrícolas y Pecuarias (INIFAP). Km 42 Carr. México-Texcoco, Apartado Postal 10. 56230, Chapingo, México. Tel. 01(595) 9521500 Ext. 5211. ${ }^{2}$ Programa de Maíz, Edo. de Hidalgo, INIFAP. Km. 3.6 Carr. PachucaCd. Sahagún, No. 200 Centro Comercial El Saucillo, Despacho 111. 42080, Mineral de la Reforma, Hgo. ${ }^{3}$ Programa de Recursos Genéticos, Campo Experimental Valle de México, INIFAP. Km 42 Carr. MéxicoTexcoco. Apartado Postal 10. 56230, Chapingo, México.
\end{abstract}

* Autor para correspondencia (gricelda vazquez@yahoo.com)

\section{RESUMEN}

En el Estado de Hidalgo, México, se establecen alrededor de 200 mil hectáreas de maíz (Zea mays L.) bajo condiciones de temporal o secano, $95 \%$ corresponde a maíces criollos. Los objetivos de esta investigación fueron determinar la calidad del grano y tortilla de 26 maíces criollos del Altiplano y Valle del Mezquital, Hidalgo, México, e identificar las razas sobresalientes para la industria de la masa y la tortilla. Dichas accesiones incluían las razas Elotes Cónicos, Chalqueño, Cónico, Tuxpeño, Arrocillo, Celaya, Tabloncillo, Ratón, Pepitilla, Cacahuacintle, Palomero Toluqueño, Olotillo, Elotes Occidentales, Cónico Norteño y Bolita. El análisis de varianza mostró diferencia significativa $(P \leq \mathbf{0 . 0 5})$ en todas las variables analizadas. Las razas preferidas por los productores fueron Cónico (32 \%), Chalqueño (20 \%) y Elotes Cónicos (18 \%). Los maíces presentaron una variedad de colores (negros, rojos, amarillos, anaranjados pintos y blancos), de tamaños (grande, mediano y pequeño) y de texturas (muy suave, suave, intermedio y duro), así como altos porcentajes de pedicelo $y$ pericarpio $y$ bajas proporciones de germen. Algunas de estas variables limitan su procesamiento en los molinos de masa-tortilla. Con las razas Cónico, Chalqueño, Tuxpeño, Cónico Norteño, Olotillo, Pepitilla y Celaya de grano blanco y textura suave e intermedia, se produjeron las tortillas de mejor calidad, con mayor: humedad $(\mathbf{4 0 . 3} \%)$, relación masa/grano (2.0:1.1) y tortillas/grano (1.4:1.1) y requirieron poca fuerza para romperse (suaves) después de haber sido elaboradas $(<180 \mathrm{gf})$, la fuerza se incremento significativamente $(P<\mathbf{0 . 0 5}), 24$ horas des-

Recibido: 09 de Febrero del 2010.

Aceptado: 21 de Junio del 2010. pués del almacenamiento a $4{ }^{\circ} \mathrm{C}(\bar{x}=191.4 \mathrm{gf})$. Asimismo, se encontró que eran de apariencia luminosa $\left(L^{*}=71.8 \%\right)$, sin observar cambios durante su almacenamiento (71.5 \%).

Palabras clave: Zea mays, criollos, dureza, relación tortilla/grano, textura de tortillas.

\section{SUMMARY}

In the state of Hidalgo, México, around 200 thousand hectares of maize (Zea mays L.) are grown under rainfed conditions; $95 \%$ of which are local landraces. This study was conducted to evaluate grain and tortilla quality of 26 maize landraces from the High Valleys and Valley of Mezquital, Hidalgo, México, and to identify outstanding races for the maize dough and tortilla industry. These accessions include the races Elotes Cónicos, Chalqueño, Cónico, Tuxpeño, Arrocillo, Celaya, Tabloncillo, Ratón, Pepitilla, Cacahuacintle, Palomero Toluqueño, Olotillo, Elotes Occidentales, Cónico Norteño and Bolita. Analysis of variance revealed significant differences $(P \leq 0.05)$ in all of the variables analyzed. Races preferred by farmers were Cónico (32 \%), Chalqueño (20 \%) and Elotes Cónicos (18 \%). There was a wide range of colors (black, red, yellow, spotted and white), sizes (large, medium and small), and textures (very soft, soft, medium and hard), as well as high percentages of pedicle and pericarp and low proportions germ. Some of these variables limit processing in maize dough-tortilla mills. The Cónico, Chalqueño, Tuxpeño, Cónico Norteño, Olotillo, Pepitilla and Celaya races, which have white grain and soft and medium texture, yielded the best quality tortillas with the highest moisture content $(\mathbf{4 0 . 3 \% )}$, the highest maize dough/kernel ratio (2.0:1.1) and the highest tortilla/kernel ratio (1.4:1.1); little force was required to break the tortillas (soft) $(<180 \mathrm{gf})$, but the required force increased

significantly $(\mathrm{P}<0.05)$ after storage for $24 \mathrm{~h}$ at $4{ }^{\circ} \mathrm{C}(\bar{x}=191.4$ gf). These tortillas had a luminous appearance $\left(\mathrm{L}^{*}=\mathbf{7 1 . 8} \%\right)$, with no observable changes during storage $(71.5 \%)$.

Index words: Zea mays, local races, hardness, tortilla/kernel ratio, tortilla texture.

\section{INTRODUCCIÓN}

En el Estado de Hidalgo, México, existen alrededor de 200 mil hectáreas sembradas con maíz (Zea mays L.) en condiciones de temporal o secano, superficie de la cual 95 $\%$ corresponde a maíces criollos. El Banco de Germoplasma del Instituto Nacional de Investigaciones Forestales, Agrícolas y Pecuarias (INIFAP), ubicado en el Campo Experimental Valle de México (CEVAMEX), cuenta con 236 colectas de maíz de ese estado, que datan de 1946 a 1972, donde predominan las razas Cónico, Chalqueño, Celaya y Elotes Cónicos (Serratos, 2009). En adición a ello, en 2004 se hicieron nuevas colectas que permitieron identificar, además, otras razas como Tuxpeño, Arrocillo, Tabloncillo, Ratón, Pepitilla, Cacahuacintle, Palomero Toluqueño, Olotillo, Elotes Occidentales, Cónico Norteño y Bolita. Esta gama de maíces criollos, aunque son de bajo rendimiento, sobresalen por su adaptabilidad a condiciones adversas, capaces de sobrevivir donde los maíces mejorados no tienen oportunidad, debido al estrés hídrico a que están expuestos y por su ciclo vegetativo de intermedio a tardío. 
Productores de las regiones temporaleras prefieren sus maíces criollos, por la adaptación a las condiciones ambientales de sus parcelas, por la facilidad para su procesamiento, por las características de los alimentos que con ellos se preparan y porque satisfacen las necesidades de su ganado (forraje), atributos que están ligados sobre todo a aspectos culturales. De manera general, la preferencia entre los distintos tipos de maíz es por atributos de color, sabor, textura, consistencia de las tortillas y facilidad de la masa para trabajarla (Pérez et $a l ., 2006)$, de igual manera la norma NMX-034 (2002) para maíz blanco destinado al proceso de nixtamalización, incluye principalmente variables de calidad del grano, entre las que destaca la dureza, así como pericarpio retenido en el nixtamal, pérdida de sólidos y humedad del nixtamal.

Los industriales de la masa y la tortilla prefieren procesar maíces de tamaño y color uniforme, optan por los de color blanco-crema brillante y con textura intermedia-dura. Ponderan los maíces con alta relación de masa/grano ( $\geq 2.0: 1.0)$ y de tortilla/maíz $(\geq 1.5 \mathrm{~kg}$ por $\mathrm{kg}$ de maíz procesado).

Finalmente, en el centro del país, los molineros no compran maíces que imparten colores grisáceos a las tortillas (Salinas et al., 2007). En tal contexto los objetivos de esta investigación fueron determinar la calidad del grano y las tortillas de 26 maíces criollos del Altiplano y Valle del Mezquital, Hidalgo, México, e identificar las razas sobresalientes para la industria de la masa y la tortilla.

\section{MATERIALES Y MÉTODOS}

A partir del ciclo primavera-verano 2004, investigadores del INIFAP colectaron maíces criollos en las zonas del Altiplano Hidalguense (2100 a 2905 msnm) y del Valle del Mezquital (1900 a $2646 \mathrm{msnm}$ ). El primero se localiza en la porción centro sureste del estado, entre las coordenadas LE: $98^{\circ} 12^{\prime}$ 00', LO: $98^{\circ} 53^{\prime} 12^{\prime}$ ', LN: $20^{\circ} 17^{\prime} 28^{\prime}$, , LS: $19^{\circ} 42^{\prime}$ 00', conformado por superficies planas a ligeramente inclinadas. Presenta clima templado subhúmedo y semifrío subhúmedo con lluvias en verano, y temperaturas entre 12 y $18{ }^{\circ} \mathrm{C}$. El Valle del Mezquital comprende la región suroeste de la entidad, entre las coordenadas LE: $98^{\circ}$ 56' $42^{\prime \prime}$, LO: $99^{\circ} 38^{\prime}$ 56', LN: $20^{\circ} 37^{\prime}$ 00', LS: $19^{\circ}$ 54' 14', con una de las superficies más planas del estado. Presenta un clima semiseco templado con lluvias en verano y una temperatura promedio anual de $17{ }^{\circ} \mathrm{C}$ (INEGI, 1992).

\section{Calidad del grano}

Se evaluaron 26 accesiones (Cuadro 1), provenientes de 23 localidades. Los análisis se realizaron en el Laboratorio de Calidad de Maíz del INIFAP. La calidad del grano se midió como peso hectolítrico, peso de 100 granos e índice de flotación como medida indirecta de la dureza, conforme a las metodologías descritas en la norma NMX-034 (2002), para efecto de comercialización del grano, se requiere que los maíces cumplan con estas especificaciones. Además, se determinaron los porcentajes de pedicelo, pericarpio y germen, de acuerdo con la metodología descrita por Salinas y Vázquez (2006). La proteína se cuantificó con el método automatizado del equipo Technicon modelo Auto Analyzer II ${ }^{\circledR}$ (modelo AutoAnalyzer, II, de manufactura norteamericana, USA), con el protocolo descrito por Wall y Gehrke (1974).

\section{Calidad de tortillas}

La calidad del nixtamal, la masa y las tortillas se evalúo, también, con los métodos descritos por Salinas y Vázquez (2006). Los tiempos de nixtamalización se asignaron de acuerdo a lo recomendado por Salinas et al., (1992), así los maíces de textura muy suave recibieron 25 min de nixtamalización, los suaves $30 \mathrm{~min}$, los intermedios $35 \mathrm{~min}$ y los duros $40 \mathrm{~min}$. En el nixtamal se registraron los porcentajes de pericarpio remanente, pérdida de sólidos y humedad. En masa se cuantificó la humedad y la relación masa/grano, que representa la cantidad de masa obtenido por kg de maíz nixtamalizado. En tortillas se determinó humedad (\%); relación tortilla/maíz, expresado como kilogramos de tortillas obtenidas por kilogramo de maíz nixtamalizado. El porcentaje de pérdida de peso durante el cocimiento de la tortilla, se calculo con la relación propuesta por Mauricio et al. (2004): \% PP = [(Peso de tortilla cruda - Peso de tortilla cocida)/(Peso de tortilla cruda) $] * 100$.

Respecto al color de las tortillas, Salinas et al. (2007) informaron que algunos maíces criollos imparten colores grises a este alimento, por lo que en esta investigación se evaluó el color de las tortillas recién elaboradas y con 24 $\mathrm{h}$ de almacenamiento en refrigeración, por ser la forma más usual de conservación. El color se midió, con el colorímetro MiniScan XE plus ${ }^{\circledR}$ (Hunter-Lab, modelo $45 / 0-\mathrm{L}$ ), se informan las variables de luminosidad $\left(\mathrm{L}^{*}\right)$ y el ángulo de tono o hue $\left(\mathrm{h}=\arctan \left(\mathrm{b} * / \mathrm{a}^{*}\right)\right.$ (Voss, 1992). La fuerza de tensión al corte de las tortillas se evaluó siguiendo la metodología descrita por Arambula et al. (2004), se uso el equipo texturómetro (Texture Analyser TA-XT2 ${ }^{\circ}$, Stable Micro Systems, England) con el accesorio AT/G que corresponde a unas pinzas de retención, en las que se sostuvo un fragmento de tortilla y 
se sometió a la fuerza de tensión. El texturómetro se calibró a una velocidad de $1 \mathrm{~mm} / \mathrm{s}$ y una distancia de 15 $\mathrm{mm}$, utilizando la celda de carga de $5 \mathrm{~kg}$. Los resultados se expresan en gramos-fuerza $\left(\mathrm{g}_{\mathrm{f}}\right)$ y representaran la media aritmética de cuatro repeticiones. Las mediciones se hicieron en tortillas atemperadas a temperatura de cuarto $\left(22{ }^{\circ} \mathrm{C} \pm 3{ }^{\circ} \mathrm{C}\right)$, a las 2 y $24 \mathrm{~h}$ después de elaboradas, estas últimas se empacaron en bolsas de polietileno, que se sellaron y se almacenaron a $4{ }^{\circ} \mathrm{C}$. A las $24 \mathrm{~h}$ se sacaron del refrigerador, se dejaron una hora al medio ambiente para evaluarlas a temperatura de cuarto, color y fuerza de tensión.

Los datos se analizaron con un modelo completamente al azar, con dos repeticiones. Se hicieron análisis de varianza, pruebas de comparación de medias de Tukey ( $\alpha$ $=0.05)$, así como la matriz de correlaciones simples, con el paquete estadístico SAS versión 9.1 (SAS Institute, 1990).

Cuadro 1. Identificación de maíces criollos colectados en el Altiplano y Valle del Mezquital, del Edo. de Hidalgo, México.

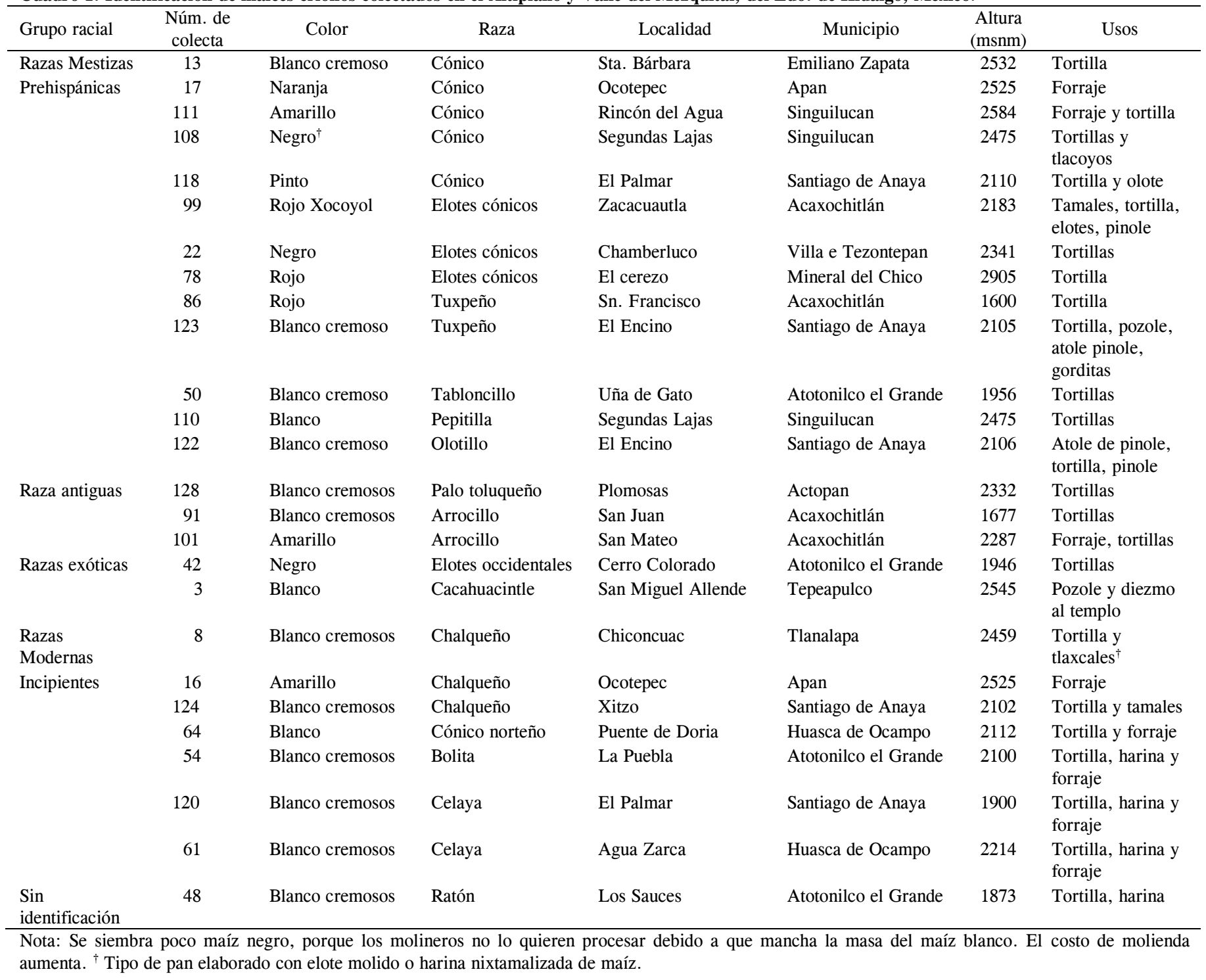




\section{RESULTADOS Y DISCUSIÓN}

\section{Calidad del grano}

El análisis estadístico mostró que había diferencia significativa $(\mathrm{P} \leq 0.05)$ al interior del grupo de colectas, en todas las variables evaluadas (Cuadro 2). Se estableció que $32 \%$ de las colectas pertenecían a la raza de maíces Cónicos, $20 \%$ a la raza Chalqueño y $18 \%$ a Elotes Cónicos, que son las razas de mayor importancia por superficie sembrada y preferencia de los productores. El restante $30 \%$ incluyó a las razas Tuxpeño, Arrocillo, Celaya, Tabloncillo, Ratón, Pepitilla, Cacahuacintle, Palomero Toluqueño, Olotillo, Elotes Occidentales, Cónico Norteño y Bolita.

La diversidad genética se manifestó en la amplia gama de colores, que fueron desde el blanco, hasta el negro, incluyendo los pintos; lo mismo que en el tamaño de sus granos, que varió desde muy grandes (Elotes Occidentales y Cacahuacintle) hasta muy pequeños (Palomero Toluqueño). En específico, la raza de maíces Cónicos incluyó colectas de grano blanco, azul, rojo, naranja, amarillo y pinto. De igual manera, la dureza varió desde granos muy suaves (19\%), suaves (23\%), intermedios (39\%) у duros (19\%). Cinco de las ocho accesiones de grano pigmentado (Cuadro 3) fueron de textura muy suave y suave, corroborando que los maíces de color, son principalmente de éste tipo de texturas (Salinas et al., 2003).
El resto de las razas fueron de textura intermedia y dura, su peso hectolítrico fue menor a lo demandado por la norma NMX-034 (2002) para maíces destinados al proceso de nixtamalización (Cuadro 3), sin embargo, con la temperatura de nixtanalización usada en la mayoría de los molinos de masa-tortilla (entre 80 y $85{ }^{\circ} \mathrm{C}$ ) y los tiempos de reposo (14 a $18 \mathrm{~h}$ ), este tipo de maíces logra una buena hidratación del grano y masas fáciles de moldear, además de resistir cierta falta de control en las variables de cantidad de cal, tiempos de nixtamalización y de reposo, así como en la molienda (Almeida-Domínguez et al., 1997; Vázquez et al., 2003).

Maíces de especialidad como Cacahuacintle y Elotes Occidentales, de textura muy suave, se usan en la elaboración de esquites ${ }^{1}$, elotes cocidos, pozole y tamales (Mauricio et al., 2004; Vázquez et al., 2003), su nixtamalización para elaborar tortilla demanda un manejo especial, pues son muy susceptibles al sobrecocimiento, por lo que los industriales de la masa y la tortilla no los procesan. A este respecto, en el área rural las amas de casa sancochan el grano de maíz, tapan el recipiente que contiene el nixtamal y lo dejan en reposo hasta que se suaviza el pericarpio. A nivel experimental se asignan tiempos cortos de nixtamalización ( $25 \mathrm{~min}$ ), con lo que se logra masa con buena textura y fácil de moldear.

Cuadro 2. Cuadrados medios de parámetros de calidad y proteína en grano y calidad de tortillas de maíces criollos del Edo. de Hidalgo, México.

\begin{tabular}{|c|c|c|c|c|c|c|c|}
\hline \multicolumn{2}{|l|}{ Fuente de variación } & \multicolumn{2}{|c|}{ Colecta } & \multirow{2}{*}{$\begin{array}{l}\text { Error } \\
26.0\end{array}$} & \multirow[t]{2}{*}{$\mathrm{R}^{2}$} & \multirow[t]{2}{*}{$\mathrm{CV}$} & \multirow[t]{2}{*}{ Media general } \\
\hline gl & & 25.0 & & & & & \\
\hline Peso de 100 granos & & 174.5 & $* *$ & 0.3 & 0.9 & 1.5 & 36.3 \\
\hline $\mathrm{PH}^{\dagger}$ & & 18.2 & $* *$ & 0.3 & 0.9 & 0.8 & 65.5 \\
\hline IF & & 1319.1 & $* *$ & 3.4 & 0.9 & 3.1 & 60.5 \\
\hline Pedicelo & & 0.4 & $* *$ & 0.1 & 0.7 & 16.6 & 2.0 \\
\hline Pericarpio & & 1.2 & $* *$ & 0.1 & 0.9 & 5.2 & 6.2 \\
\hline Germen & & 1.4 & $* *$ & 0.5 & 0.7 & 6.7 & 10.3 \\
\hline $\mathrm{PR}$ & & 395.7 & $* *$ & 36.2 & 0.9 & 16.9 & 35.6 \\
\hline HT & & 21.4 & $* *$ & 3.8 & 0.8 & 5.0 & 38.7 \\
\hline $\mathrm{R} \mathrm{T} / \mathrm{M}$ & & 0.02 & $* *$ & 0.0 & 1.0 & 0.0 & 1.2 \\
\hline \multirow[b]{2}{*}{ Luminosidad (L*) } & $2 \mathrm{~h}$ & 253.0 & $* *$ & 0.1 & 0.9 & 0.2 & 64.9 \\
\hline & $24 \mathrm{~h}$ & 254.0 & $* *$ & 0.1 & 0.9 & 0.6 & 64.4 \\
\hline \multirow{2}{*}{ Tono (Hue) } & $2 \mathrm{~h}$ & 986.5 & $* *$ & 7.0 & 0.9 & 2.8 & 93.4 \\
\hline & $24 \mathrm{~h}$ & 1045.7 & $* *$ & 17.9 & 0.9 & 4.5 & 93.5 \\
\hline \multirow{2}{*}{ Fuerza $g_{f}$} & $2 \mathrm{~h}$ & 5642.0 & $* *$ & 10.2 & 0.9 & 1.7 & 189.9 \\
\hline & $24 \mathrm{~h}$ & 6763.0 & $* *$ & 24.6 & 0.9 & 2.7 & 182.8 \\
\hline
\end{tabular}

*, ** Diferente de cero a una probabilidad de 0.05 y 0.01 , respectivamente. gl $=$ grados de libertad; $\mathrm{R}^{2}=$ coeficiente de determinación; $\mathrm{CV}=$ coeficiente de variación. ${ }^{\dagger} \mathrm{PH}=$ peso hectolítrico; $\mathrm{IF}=$ índice de flotación; $\mathrm{PR}=$ percarpio retenido; HT $=$ humedad en tortillas; $\mathrm{R}$ T/M $=$ relación tortilla/maíz; $\mathrm{g}_{\mathrm{f}}=$ gramos fuerza.

\footnotetext{
${ }^{1}$ Granos tiernos de maíz, fritos en aceite y aderezados con cebolla, chile
} y epazote. 
Las razas con mayores porcentajes de pedicelo y pericarpio fueron Elotes Cónicos, Cónico Norteño, Palomero Toluqueño y Pepitilla, con niveles superiores a lo indicado en la literatura para maíces dentados (González, 1995), inversamente registraron un reducido porcentaje de germen (Cuadro 3). Para los industriales de la masa y la tortilla, los altos porcentajes de pericarpio, no representa problema en la producción de tortillas de colores claros y brillantes, siempre que el pericarpio sea blanco y sin colores indeseables que pudieron ser impartidos por condiciones climáticas, ataque de hongos, insectos u otros daños. En trabajos previos (Vázquez et al., 1990; Vázquez et al., 2003) la raza Pepitilla también registró elevado porcentaje de pericarpio, lo que pudiera estar relacionado con la buena textura de sus tortillas
(Vázquez et al., 2003). El aprovechamiento del maíz Cacahuacintle como elote o esquites pudiera explicarse por su reducido porcentaje de pericarpio (Cuadro 3), por lo que resulta más agradable al paladar en su estado tierno.

El mayor porcentaje de germen correspondió a las razas Chalqueño y Elotes Cónicos (amarillo y negro, respectivamente) (Cuadro 3). Normalmente esto se relaciona con un mayor contenido de aceite en el grano, lo que contribuye a una mejor textura de las tortillas y una mejor nutrición para los consumidores, debido a la cantidad de ácidos grasos insaturados y niveles elevados de antioxidantes naturales (Bressani, 2008).

Cuadro 3. Variables de calidad del grano y proteína de maíces criollos del Edo. de Hidalgo, México.

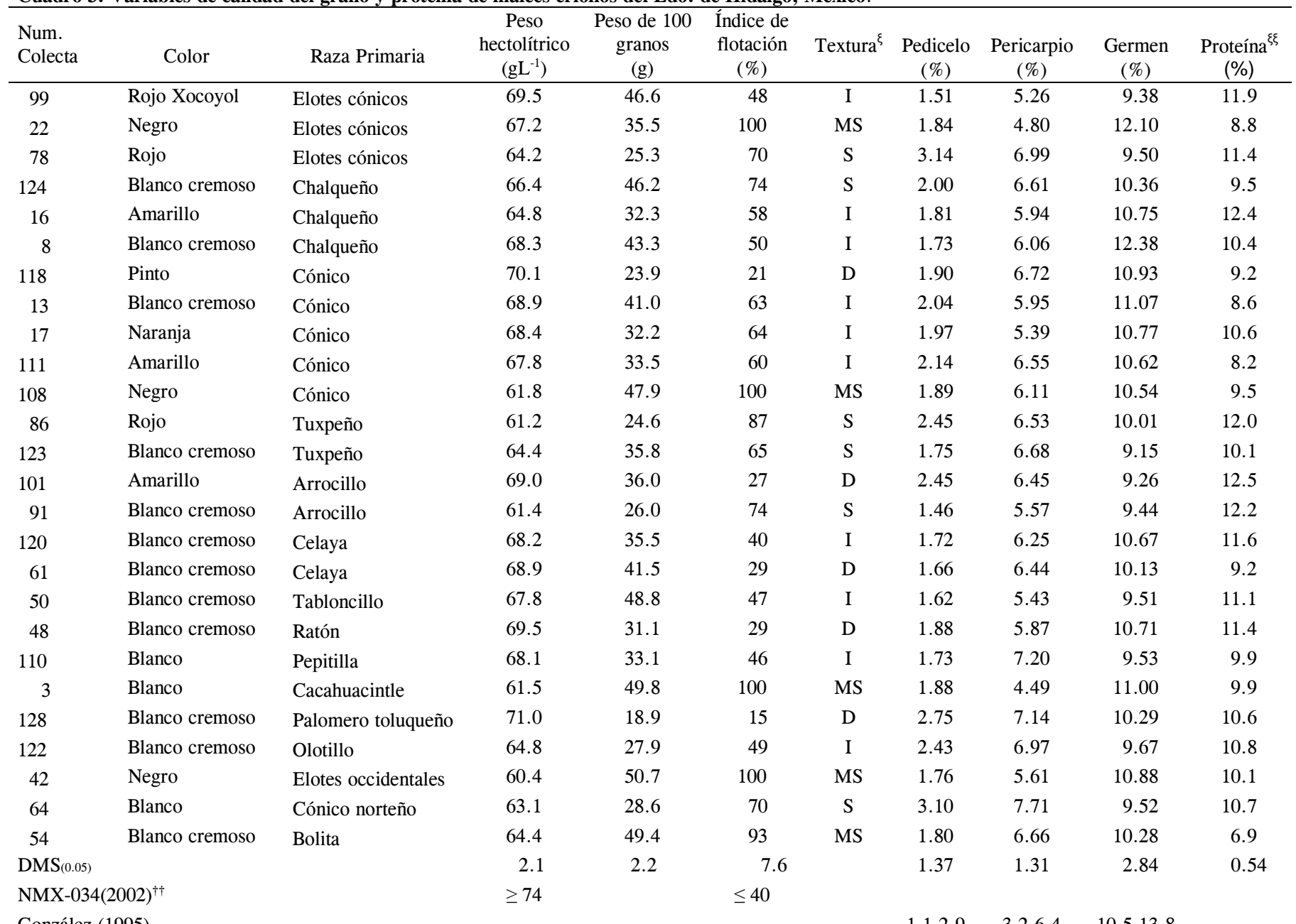

$1.1-2.9 \quad 3.2-6.4 \quad 10.5-13.8$

DMS = diferencia mínima significativa. $\dagger \dagger$ Valores establecidos por la norma mexicana para maíz nixtamalizado (NMX-FF-034-2002-SCF1/P-1). $\xi \xi$ N x 6.25, expresado en base seca. $\xi$ Índices de Flotación entre 0 a $12 \%=$ granos muy duros; 13 a $37 \%=$ duros; 38 a $62 \%=$ intermedios; 63 a $87 \%=$ suaves; más de $88 \%$ = muy suave. 
El contenido de proteína varió de 6.9 a $12.5 \%$ (Cuadro 3) con un valor medio de $10.4 \%$, los resultados estuvieron dentro de lo reportado para maíces criollos (Vázquez et al., 2003). Los maíces criollos del Estado de Hidalgo se caracterizaron por presentar una variedad de colores (desde negros hasta blancos), tamaños (de grandes a pequeños) y dureza (muy suave hasta duros), así como altos porcentajes de pedicelo y pericarpio, y baja proporción de germen. Algunas de estas variables limitan su procesamiento en la industria de la masa y la tortilla. Se identificaron ocho razas de grano blanco y textura intermedia-dura (Chalqueño colecta 8, Cónico 13, Arrocillo 101, Celaya 120, Tabloncillo 50, Ratón 48, Pepitilla 110 y Olotón 122) que pueden ser procesadas por los industriales de la masa y la tortilla.

\section{Calidad de tortillas}

El $82 \%$ de los maíces criollos retuvieron menos de 50 $\%$ de pericarpio en el nixtamal, lo que significa que su pericarpio se hidrolizó fácilmente durante la nixtamalización, no obstante, las pérdidas de sólidos $(\bar{x}=$ $3.4 \%$ ) fueron menores a lo establecido como máximo en la norma NMX-034 (2002) (Cuadro 4). Razas de usos especiales como, Palomero Toluqueño (colecta 128) y Cacahuacintle (3), además Cónico (17), registraron las mayores pérdidas de sólidos (Cuadro 4). La humedad del nixtamal varió de 36 a $55 \%$ y fue superior a lo establecido en la norma NMX-034 (2002) (Cuadro 4). Las colectas con menor peso de 100 granos, y de acuerdo con Billeb y Bressani (2001) también de menor tamaño, registraron la mayor humedad en nixtamal $(r=-0.45 * *)$, masa $(r=-0.62 * *)$ y tortillas $(r=-0.67 * *)$. La humedad en la masa fue alta, lo que se atribuye a una mayor absorción de agua por parte de los maíces de textura suave, como lo informaron Salinas et al. (2003), de igual manera, las pérdidas de peso por efecto del cocimiento de la masa fueron altas (34 \%, datos no mostrados), pero estuvieron dentro de lo informado para maíces criollos (Mauricio et al., 2004).

Con las razas de grano muy suave: Elotes Cónicos (colecta 22), Cacahuacintle (3), Elotes Occidentales (42), Bolita (54) y Cónico (108), se produjeron tortillas con menor humedad, debido a una mayor pérdida de peso durante el cocimiento de la masa (39 \%), consecuentemente su relación tortilla/maíz estuvo entre las más bajas (1.3:1.0). En las colectas 22, 3, 42, y 54 las tortillas recién elaboradas requirieron más fuerza para romperse, que las almacenadas durante $24 \mathrm{~h}$ (Cuadro 4), característica que pudiera deberse a la retrogradación del almidón, especialmente de la amilasa, que retrograda más rápido que la amilopectina (Rooney y Suhendro, 1999), por otra parte, el cambio de temperatura de cuarto, a 4
${ }^{\circ} \mathrm{C}$, pudo provocar condensación del agua en el empaque, y ésta inducir una mayor suavidad en las tortillas a las 24 h (Cuadro 4).

El nixtamal del maíz Cacahuacintle, retuvo el menor porcentaje de pericarpio (Cuadro 4), lo que facilitó su remoción durante el lavado y fue de los materiales que más sólidos desprendieron (4\%). Estas características han convertido a esta raza en un maíz de especialidad, demandado, como ya se ha mencionado, para consumirse en forma de pozole o bien como elote (Bonifacio et al., 2005; Narváez-González et al., 2007).

Un caso diferente fue la colecta 78 de Elotes Cónicos, de grano rojo, que presento un $7 \%$ de pericarpio en el grano y retuvo $82 \%$ en su nixtamal, consecuentemente, su pérdida de sólidos fue reducida $(2.8 \%)$, lo que constituyó una característica única para esta accesión (78). Parte del pigmento rojo se solubilizó en el nejayote y el pericarpio adherido al grano nixtamalizado, le impartió un color desagradable a la masa, y consecuentemente a la tortilla, cuya luminosidad $\left(\mathrm{L}^{*}=41.4 \%\right)$ y ángulo de tono $\left(\mathrm{h}=68^{\circ}\right)$, fueron los más bajos del grupo. Estas características pudieran estar asociadas a las bajas temperaturas donde se produjo, consecuencia de la altura (2905 msnm) (Cuadro 1), así como mayor intensidad de la radiación solar, que de acuerdo con Muñoz (2009; Com. personal $\mathrm{l}^{2}$ ) inducen una mayor síntesis de pigmentos.

La asignación de tiempos de nixtamalización de acuerdo con la dureza del grano, permitió elaborar tortillas de buena calidad a partir de maíces de textura suave e intermedia, tal fue el caso de las razas Cónico (13), Chalqueño (124 y 16), Tuxpeño (123), Cónico Norteño (64), Pepitilla (110) y Celaya (120), las cuales tuvieron buena humedad $(40.3 \%)$, registraron la mayor relación de masa/grano (1.97:1.1) y de tortillas/maíz (1.4:1.1) y fueron las de textura más suaves después de haber sido elaboradas $\left(<180 \mathrm{~g}_{\mathrm{f}}\right)$, la fuerza requerida para romperlas se incremento significativamente $(\mathrm{P}<0.05), 24 \mathrm{~h}$ después del almacenamiento a $4{ }^{\circ} \mathrm{C}\left(\bar{x}=191.4 \mathrm{~g}_{\mathrm{f}}\right)$. Asimismo, se detectaron de apariencia luminosa $\left(\mathrm{L}^{*}=\right.$ $71.8 \%)$ y tono crema $\left(\mathrm{h}=87.1^{\circ}\right)$, sin observar cambios durante su almacenamiento $\left(71.5 \%\right.$ y $87.6^{\circ}$, respectivamente). La metodología de evaluación permitió corroborar que con las razas de mayor preferencia por los productores (Cónico y Chalqueño) se elaboraron tortillas de buena calidad, lo mismo que las de Elotes Cónicos (78) que fueron de color café (Cuadro 4) y que a nivel rural son aceptadas.

\footnotetext{
${ }^{2}$ Abel Muñoz Orozco, Profesor-investigador del Instituto de Recursos Genéticos, del Colegio de Postgraduados. Montecillo, Edo de México.
} 
Cuadro 4. Variables de calidad de nixtamal, masa y tortillas de maíces criollos del Edo. de Hidalgo, México.

\begin{tabular}{|c|c|c|c|c|c|c|c|c|c|c|c|c|c|}
\hline \multirow{2}{*}{$\begin{array}{l}\text { Num. de } \\
\text { colecta }\end{array}$} & \multirow{2}{*}{ Color } & \multirow{2}{*}{ Raza Primaria } & \multirow{2}{*}{$\begin{array}{l}\mathrm{PR}^{\dagger \dagger} \\
(\%)\end{array}$} & \multirow{2}{*}{$\begin{array}{c}\text { Sólidos } \\
(\%)\end{array}$} & \multicolumn{3}{|c|}{ Humedad (\%) } & \multicolumn{2}{|c|}{ Índice } & \multicolumn{2}{|c|}{ Luminmosidad (\%) } & \multicolumn{2}{|c|}{ Fuerza $\left(g_{f}\right)$} \\
\hline & & & & & Nixtamal & Masa & Tortilla & Masa & Tortilla & $2 \mathrm{~h}$ & $24 \mathrm{~h}$ & $2 \mathrm{~h}$ & $24 \mathrm{~h}$ \\
\hline 99 & Rojo Xocoyol & Elotes cónicos & 45.6 & 2.4 & 35.8 & 53.9 & 36.3 & 1.8 & 1.3 & 46.6 & 47.0 & 162.8 & 146.8 \\
\hline 22 & Negro & Elotes cónicos & 32.7 & 3.3 & 46.3 & 57.0 & 35.4 & 1.9 & 1.2 & 44.4 & 43.9 & 256.8 & 213.2 \\
\hline 78 & Rojo & Elotes cónicos & 81.5 & 2.8 & 45.7 & 58.8 & 40.8 & 2.1 & 1.4 & 41.4 & 40.0 & 149.8 & 181.8 \\
\hline 124 & Blanco cremoso & Chalqueño & 35.9 & 3.7 & 49.0 & 59.0 & 41.0 & 2.2 & 1.4 & 72.3 & 73.5 & 155.3 & 159.3 \\
\hline 16 & Blanco cremoso & Chalqueño & 20.5 & 3.6 & 48.6 & 56.0 & 38.0 & 1.9 & 1.3 & 74.3 & 71.4 & 254.8 & 149.5 \\
\hline 8 & Amarillo & Chalqueño & 31.0 & 3.8 & 45.6 & 56.3 & 37.9 & 1.9 & 1.3 & 70.5 & 69.4 & 188.3 & 147.8 \\
\hline 118 & Pinto & Cónico & 42.1 & 3.4 & 48.1 & 58.1 & 43.6 & 1.8 & 1.4 & 59.7 & 59.3 & 206.9 & 156.5 \\
\hline 13 & Blanco cremoso & Cónico & 20.4 & 3.6 & 45.7 & 57.1 & 37.9 & 2.0 & 1.3 & 73.3 & 72.4 & 131.5 & 261.6 \\
\hline 17 & Naranja & Cónico & 26.1 & 4.4 & 45.8 & 57.3 & 36.7 & 2.0 & 1.3 & 62.3 & 61.3 & 126.3 & 244.0 \\
\hline 111 & Amarillo & Cónico & 21.7 & 4.0 & 46.8 & 57.0 & 41.1 & 1.8 & 1.3 & 62.3 & 61.3 & 128.2 & 145.0 \\
\hline 108 & Negro & Cónico & 32.8 & 2.9 & 47.2 & 56.2 & 35.2 & 2.0 & 1.3 & 43.6 & 43.2 & 162.7 & 183.8 \\
\hline 86 & Rojo & Tuxpeño & 26.4 & 3.1 & 55.9 & 59.8 & 42.7 & 1.9 & 1.2 & 53.9 & 53.8 & 294.5 & 173.6 \\
\hline 123 & Blanco cremoso & Tuxpeño & 39.1 & 3.0 & 45.1 & 58.5 & 40.4 & 2.2 & 1.5 & 69.9 & 70.1 & 157.9 & 164.6 \\
\hline 101 & Blanco cremoso & Arrocillo & 39.4 & 3.4 & 47.8 & 59.4 & 45.2 & 1.9 & 1.3 & 69.3 & 69.2 & 137.1 & 129.6 \\
\hline 91 & Amarillo & Arrocillo & 25.6 & 3.2 & 40.8 & 56.2 & 37.7 & 2.0 & 1.4 & 67.4 & 67.2 & 133.8 & 184.4 \\
\hline 120 & Blanco cremoso & Celaya & 29.8 & 3.5 & 45.2 & 58.8 & 41.9 & 2.1 & 1.4 & 74.9 & 73.7 & 179.6 & 145.7 \\
\hline 61 & Blanco cremoso & Celaya & 35.4 & 3.3 & 41.0 & 58.1 & 39.7 & 2.1 & 1.4 & 70.9 & 70.9 & 183.7 & 175.4 \\
\hline 50 & Blanco cremoso & Tabloncillo & 31.0 & 3.1 & 38.2 & 55.8 & 36.1 & 1.8 & 1.3 & 71.7 & 70.8 & 255.0 & 155.9 \\
\hline 48 & Blanco cremoso & Ratón & 23.3 & 3.5 & 44.4 & 58.5 & 37.0 & 1.8 & 1.3 & 70.3 & 68.5 & 277.2 & 378.8 \\
\hline 110 & Blanco & Pepitilla & 57.0 & 3.3 & 47.4 & 61.6 & 39.5 & 2.1 & 1.4 & 71.4 & 70.4 & 167.4 & 262.8 \\
\hline 3 & Blanco & Cacahuacintle & 18.8 & 4.0 & 47.2 & 54.9 & 30.0 & 1.7 & 1.2 & 74.8 & 75.2 & 233.9 & 226.0 \\
\hline 128 & Blanco cremoso & Palomero toluqueño & 39.2 & 4.4 & 51.0 & 59.8 & 40.3 & 2.1 & 1.4 & 71.8 & 72.7 & 164.1 & 163.6 \\
\hline 122 & Blanco cremoso & Olotillo & 58.0 & 3.2 & 52.0 & 59.2 & 41.5 & 2.1 & 1.4 & 71.8 & 72.9 & 206.0 & 172.1 \\
\hline 42 & Negro & Elotes occidentales & 32.8 & 3.5 & 54.7 & 59.1 & 38.2 & 1.8 & 1.3 & 46.1 & 45.4 & 187.3 & 170.5 \\
\hline 64 & Blanco & Cónico norteño & 49.7 & 3.7 & 55.0 & 59.4 & 39.8 & 2.2 & 1.4 & 68.1 & 68.4 & 144.1 & 234.0 \\
\hline 54 & Blanco cremoso & Bolita & 28.8 & 3.3 & 37.5 & 58.3 & 35.8 & 1.9 & 1.3 & 72.5 & 71.1 & 292.9 & 148.1 \\
\hline \multirow{2}{*}{\multicolumn{2}{|c|}{$\begin{array}{l}\operatorname{DMS}_{(0.05) \dagger} \\
\text { NMX-034(2002) }\end{array}$}} & & 24.6 & 1.1 & 12.2 & 1.4 & 7.9 & 0.25 & 0.19 & 0.5 & 1.5 & 13.0 & 20.3 \\
\hline & & & & $\leq 5.0$ & $36-42$ & & & & & & & & \\
\hline
\end{tabular}

${ }^{\dagger} \mathrm{DMS}=$ diferencia mínima significativa; ${ }^{\dagger \dagger} \mathrm{PR}=$ pericarpio retenido; ${ }^{\top}$ Cantidad de masa o tortillas por kg de maíz nixtamalizado; $\mathrm{g}_{\mathrm{f}}=$ gramos fuerza.

Las tortillas de los maíces negros (colectas 22, 108 y 42) fueron menos húmedas $(36.2 \%)$ y requirieron mayor fuerza de tensión para romperse (Cuadro 4). Las tortillas de la colecta 22 fueron duras $\left(256.8 \mathrm{~g}_{\mathrm{f}}\right)$, en tanto que las de la colecta 42 fueron de dureza media $\left(187.3 \mathrm{~g}_{\mathrm{f}}\right)$. También fueron las menos luminosas (43.61 a $46.1 \%)$ y las de mayor ángulo de tono $\left(320\right.$ a $\left.335^{\circ}\right)$. Destacaron las tortillas del maíz Cónico (108), por haber registrado mayor ángulo de tono $\left(335^{\circ}\right), 24 \mathrm{~h}$ después de elaboradas $\left(348^{\circ}\right)$, lo cual se atribuye a las modificaciones en el patrón de las antocianinas, que son los pigmentos responsables del color de los maíces negros y rojos, los cuales, según Salinas-Moreno et al. (2003), se modifican durante la nixtamalización, debido al elevado $\mathrm{pH}$.

En el bloque de los maíces de grano blanco-cremoso, el promedio de luminosidad, en tortillas de $2 \mathrm{~h}$, fue de 71.8 $\%$, en tanto que hue fue de $82.5^{\circ}$, incrementándose ligeramente en tortillas de $24 \mathrm{~h}\left(71.4 \%\right.$ y $88.7^{\circ}$, respectivamente), sobresalieron las tortillas de las razas Celaya (colecta 120) y Cacahuacintle, por haber sido las más luminosas a las dos y $24 \mathrm{~h}$ de elaboradas (Cuadro 4). Las tortillas de los maíces criollos del Estado de Hidalgo,
México, no presentaron tonos grisáceos después de $24 \mathrm{~h}$ de almacenamiento.

\section{BIBLIOGRAFÍA}

Almeida-Dominguez H D, E L Suhendro, L W Rooney (1997) Corn alkaline cooking properties related to grain characteristics and viscosity (RUA). J. Food Sci. 62:516-519.

Arámbula-Villa G, J A Méndez-Albores, J González-Hernández, E Gutiérrez-Arias, E Moreno-Martínez (2004) Evaluación de una metodología para determinar características de textura de tortilla de maíz (Zea mays L.). Arch. Latinoam. Nutr. 54:216222.

Billeb A C y R Bressani (2001) Características de cocción por nixtamalización de once variedades de maíz. Arch. Latinoam. Nutr. 51:86-94.

Bonifacio V E I, Y Salinas M, A Ramos R, A Carrillo O (2005) Calidad pozolera en colectas de maíz Cacahuacintle. Rev. Fitotec. Mex. 28:253-260.

Bressani R (2008) Cambios nutrimentales en el maíz inducidos por el proceso de nixtamalización. In: Nixtamalización del Maíz a la Tortilla. Aspectos Nutrimentales y Toxicológicos. M E Rodríguez G, S O Serna S, F Sánchez S (eds). Universidad Autónoma de Querétaro. México. pp:19-80.

González A U (1995) El Maíz y su Conservación. México. Ed.Trillas. $399 \mathrm{p}$. 
INEGI (1992) Síntesis geográfica del estado de Hidalgo. Geografía e informática (México) Instituto Nacional de Estadística. México, D. F. 134 p.

Mauricio S R A, J de D Figueroa C, S Taba, M de la L Reyes V, F Rincón S, A Mendoza G (2004) Caracterización de accesiones de maíz por calidad del grano y tortillas. Rev. Fitotec. Mex 27:213-222.

Narváez-González E D, J de D Figueroa-Cárdenas, S Taba (2007) Aspectos microestructurales y posibles usos del maíz de acuerdo con su origen geográfico. Rev. Fitotec. Mex. 30:321325.

Norma Mexicana para Maíces Destinados al Proceso de Nixtamalización, NMX-FF034-2002-SCFI-Parte-1 (2002) Productos alimenticios no industrializados-para consumo humano-cereales-Maíz blanco para proceso alcalino para tortillas de maíz y productos de maíz nixtamalizadoEspecificaciones y métodos de prueba. Secretaria de Agricultura, Ganadería, Desarrollo Rural, Pesca y Alimentación; Dirección General de Normas. México, D. F. 18 $\mathrm{p}$.

Pérez C J P, Y I Beltrán V, J M Hernández C (2006) Avances en el rescate y conservación del maíz criollo en el estado de Hidalgo. Exploración etnobotánica e investigación agronómica. In: Investigación, Docencia y Patrimonio. Memorias de las Jornadas de Arqueología. Consejo Nacional para la Cultura y las Artes - Instituto Nacional de Antropología e Historia. México, D.F. pp: 103-113.

Rooney L W, E L Suhendro (1999) Perspectives on nixtamalization (alkaline cooking) of maize for tortillas and snacks. Cereal Foods World 44:466-470.
Salinas-Moreno Y, J de J López-Reynoso, G B González-Flores, G Vázquez-Carrillo (2007) Compuestos fenólicos del grano de maíz y su relación con el oscurecimiento de masa y tortilla. Agrociencia 41:295-305.

Salinas-Moreno Y, F Martínez-Bustos, M Soto-Hernández, $\mathbf{R}$ Ortega-Paczka, J L Arellano-Vázquez (2003) Efecto de la nixtamalización sobre la antocianinas del grano de maíces pigmentados. Agrociencia 37:617-628.

Salinas M Y, F Martínez B, J Gómez H (1992) Comparación de métodos para medir la dureza del maíz (Zea mays L.) Arch. Latinoam. Nutr. 42:59-63.

Salinas M Y, G Vázquez C (2006) Metodologías de Análisis de la Calidad Nixtamalero-Tortillera en Maíz. Folleto Técnico No. 24. Instituto Nacional de Investigaciones Forestales, Agrícolas y Pecuarias. Campo Experimental Valle de México. Chapingo, Edo de México. México. $91 \mathrm{p}$.

Serratos H J A (2009) El Origen y la Diversidad del Maíz en el Continente Americano. Ed. Greenpeace. México, D. F. 33 p.

Statistical Analysis System (1990) SAS/STAT User's guide. Ver. 9.1 SAS Institute Inc. Cary, North Caroline, USA. 1686 p.

Vázquez C M G, L Guzmán B, J L Andrés G, F Márquez S (2003) Calidad de grano y tortillas de maíces criollos y sus retrocruzas. Rev. Fitotec. Mex. 26:231-238.

Vázquez-Carrillo M G, A R Márquez S, F Márquez S (1990) Evaluación física, química y tortillera del compuesto pepitilla de maíz. Rev. Fitotec. Mex. 13:117-128.

Voss D H (1992) Relating colorimeter measurement of plant color to the Royal Horticultural Society Colour Chart. HortScience 27:1256-1260

Wall L L, W Ch Gehrke (1974) Total protein measure automated Technicon BD/AAII. Method presented at the 88th Annual Meeting of the AOAC. $50 \mathrm{p}$. 